University of Wisconsin - Madison

MADPH-99-1110

AMES-HET-99-04

April 1999

\title{
Majorana neutrino masses from neutrinoless double beta decay and cosmology
}

\author{
V. Barger ${ }^{1}$ and K. Whisnant ${ }^{2}$ \\ ${ }^{1}$ Department of Physics, University of Wisconsin, Madison, WI 53706, USA \\ ${ }^{2}$ Department of Physics and Astronomy, Iowa State University, Ames, IA 50011, USA
}

\begin{abstract}
When three Majorana neutrinos describe the solar and atmospheric neutrino data via oscillations, a nonzero measurement of neutrinoless double beta $(0 \nu \beta \beta)$ decay can determine the sum of neutrino masses $\sum m_{\nu}$ if the solar solution has small-angle mixing, and place a lower bound on $\sum m_{\nu}$ for large-angle solar mixing. If in addition a nonzero $\sum m_{\nu}$ is deduced from cosmology, the neutrino mass spectrum may be uniquely specified for some ranges of neutrino parameters. For $\sum m_{\nu}>0.75 \mathrm{eV}$, the small-angle solar solution is excluded by the current upper limit on neutrinoless double beta decay. In models with maximal solar mixing the $C P$ phases of the neutrinos may be strongly constrained by stringent upper bounds on $0 \nu \beta \beta$ decay.
\end{abstract}


1. Introduction. Recent results from Super-Kamiokande [1, 2] support neutrino oscillation explanations of the solar [3, [4] and atmospheric [5, 6, 7] neutrino anomalies. Global fits to all the data indicate that neutrino oscillations among three neutrino species are sufficient to describe the solar and atmospheric data [8, 9], and estimates have been obtained for the neutrino mass-squared differences required to explain the data $\left(\delta m_{a t m}^{2} \sim 3 \times 10^{-3} \mathrm{eV}^{2}\right.$ for atmospheric neutrinos [2] and $\delta m_{\text {sun }}^{2} \sim 10^{-10}$ [9, [10, [1] or $10^{-5} \mathrm{eV}^{2}$ [10] for solar neutrinos). However, neutrino oscillations only put restrictions on the mass-squared differences, and do not constrain the absolute neutrino mass scale; they also do not distinguish whether the smaller mass splitting (the one responsible for solar neutrino oscillations) is between the two largest mass eigenstates or the two smallest. In order to learn about the actual neutrino masses, we must look elsewhere.

Studies of the power spectra of the cosmic microwave background radiation and galaxies can provide information on the sum of the neutrino masses, $\sum m_{\nu}$ [12, 13, 14]. Another possibility for learning about neutrino masses is neutrinoless double beta $(0 \nu \beta \beta)$ decay [15], which can occur if massive neutrinos are Majorana (as is nominally expected [16]), in which case lepton number is not conserved. If three Majorana neutrinos are nearly degenerate, a nonzero measurement of neutrino mass coupled with an upper limit on $0 \nu \beta \beta$ decay can place strong constraints on the Majorana neutrino mixing matrix [17]. In this Letter, we derive a simple formula that summarizes these constraints, and generalize the argument to include the effects of mass splittings. We find that a nonzero measurement of neutrinoless double beta $(0 \nu \beta \beta)$ decay would determine the sum of neutrino masses $\sum m_{\nu}$ if the solar solution has small-angle mixing, and place a lower bound on $\sum m_{\nu}$ for large-angle solar mixing. For $\sum m_{\nu}>0.75 \mathrm{eV}$, the small-angle solar solution is excluded by the current upper limit on neutrinoless double beta decay. Simultaneous nonzero measurements of $0 \nu \beta \beta$ decay and $\sum m_{\nu}$ may uniquely specify the structure of the neutrino mass eigenstates for some ranges of neutrino mass parameters. Furthermore, in models with maximal solar mixing the $C P$ phases of the neutrinos may be strongly constrained by stringent upper bounds on $0 \nu \beta \beta$ decay.

2. Formalism. We assume that there are only three active neutrino flavors with Majorana masses, and that neutrino oscillations account for the solar and atmospheric anomalies, with mass-squared differences $\delta m_{\text {sun }}^{2} \ll \delta m_{\text {atm }}^{2}$ (our arguments hold for either matterenhanced [18] or vacuum solar [19] solutions). Assigning the mass eigenvalues $m_{1}<m_{2}<m_{3}$, there are two possible mass spectra that can describe the oscillation data (see Fig. (1)

$$
\begin{array}{lll}
\delta m_{21}^{2}=\delta m_{\text {sun }}^{2}, & \delta m_{32}^{2}=\delta m_{\text {atm }}^{2}, & \text { Spectrum I } \\
\delta m_{21}^{2}=\delta m_{\text {atm }}^{2}, & \delta m_{32}^{2}=\delta m_{\text {sun }}^{2}, & \text { Spectrum II }
\end{array}
$$

where $\delta m_{j k}^{2} \equiv m_{j}^{2}-m_{k}^{2}$. Spectrum I (II) corresponds to the case where the two closely degenerate states responsible for the solar oscillation are the two smallest (largest) mass eigenstates. 
The charged-current eigenstates are related to the mass eigenstates by a unitary transformation. In Spectrum I we parametrize this transformation as

$$
\left(\begin{array}{c}
\nu_{e} \\
\nu_{\mu} \\
\nu_{\tau}
\end{array}\right)=U V\left(\begin{array}{c}
\nu_{1} \\
\nu_{2} \\
\nu_{3}
\end{array}\right)=\left(\begin{array}{ccc}
c_{1} c_{3} & c_{1} s_{3} & s_{1} e^{-i \delta} \\
-c_{2} s_{3}-s_{1} s_{2} c_{3} e^{i \delta} & c_{2} c_{3}-s_{1} s_{2} s_{3} e^{i \delta} & c_{1} s_{2} \\
s_{2} s_{3}-s_{1} c_{2} c_{3} e^{i \delta} & -s_{2} c_{3}-s_{1} c_{2} s_{3} e^{i \delta} & c_{1} c_{2}
\end{array}\right) V\left(\begin{array}{c}
\nu_{1} \\
\nu_{2} \\
\nu_{3}
\end{array}\right),
$$

where $c_{j} \equiv \cos \theta_{j}, s_{j} \equiv \sin \theta_{j}$, and $V$ is the diagonal matrix $\left(1, e^{i \phi_{2}}, e^{i\left(\phi_{3}+\delta\right)}\right)$. In Eq. (3), $\phi_{2}$ and $\phi_{3}$ are additional phases for Majorana neutrinos that are not measurable in neutrino oscillations; if $C P$ is conserved, the phases in $U V$ are either 0 or $\pi$. Then in atmospheric and long-baseline experiments, the vacuum oscillation probabilities are

$$
P\left(\nu_{\alpha} \rightarrow \nu_{\beta}\right)=4\left|U_{\alpha 3}\right|^{2}\left|U_{\beta 3}\right|^{2} \sin ^{2} \Delta_{a t m}, \quad \alpha \neq \beta
$$

where $\Delta_{a t m} \equiv 1.27\left(\delta m_{a t m}^{2} / \mathrm{eV}^{2}\right)(L / \mathrm{km}) /(E / \mathrm{GeV})$ and terms involving the solar oscillation can be ignored since they have not had time to develop. The solar $\nu_{e}$ vacuum oscillation probability is approximately given by

$$
P\left(\nu_{e} \rightarrow \nu_{e}\right)=1-2 s_{1}^{2} c_{1}^{2}-4 c_{1}^{4} s_{3}^{2} c_{3}^{2} \sin ^{2} \Delta_{\text {sun }},
$$

where $\Delta_{\text {sun }}$ is defined similarly to $\Delta_{\text {atm }}$ and the oscillations involving $\Delta_{\text {atm }}$ have averaged: $\sin ^{2} \Delta_{a t m} \rightarrow \frac{1}{2}$. The CHOOZ reactor experiment [20] imposes the constraint

$$
s_{1} \lesssim 0.23 \text { for } \delta m_{a t m}^{2}>2 \times 10^{-3} \mathrm{eV}^{2}
$$

but gives no limit for $\delta m_{\text {atm }}^{2}<10^{-3} \mathrm{eV}^{2}$. The 504-day atmospheric neutrino data imply [9]

$$
s_{1}<0.3 \text { for any } \delta m_{a t m}^{2} \text {. }
$$

Thus in Spectrum I there is very little mixing of $\nu_{e}$ with the heaviest state; because of the small size of $s_{1}^{2}$, most of the solar $\nu_{e}$ depletion is due to the $\Delta_{\text {sun }}$ term for either matterenhanced or vacuum solar neutrino oscillations, and the fitted values for the solar oscillation amplitude are not greatly affected by the particular value of $s_{1}$ [8, 9].

For Spectrum II, the oscillation probabilities can be obtained simply by interchanging the roles of $m_{1}$ and $m_{3}$. Then if $U V$ is obtained from Eq. (3) by interchange of the first and third columns of $U V$, fits to oscillation data for Spectrum II will give the same values for the parameters $\theta_{j}$ and $\phi_{k}$ as those for I. The limit on $s_{1}$ then implies that there is very little mixing of $\nu_{e}$ with the lightest state in II.

In $0 \nu \beta \beta$ decay, the decay rate depends on the $\nu_{e}-\nu_{e}$ element of the neutrino mass matrix [15], which is

$$
\begin{array}{rlr}
M_{e e} & =c_{1}^{2} c_{3}^{2} m_{1}+c_{1}^{2} s_{3}^{2} m_{2} e^{i \phi_{2}}+s_{1}^{2} m_{3} e^{i \phi_{3}}, \quad \text { (I) } \\
& =c_{1}^{2} c_{3}^{2} m_{3}+c_{1}^{2} s_{3}^{2} m_{2} e^{i \phi_{2}}+s_{1}^{2} m_{1} e^{i \phi_{3}}, \quad \text { (II) } .
\end{array}
$$

The form of $M_{e e}$ necessarily implies

$$
\left|M_{e e}\right| \leq m_{3}
$$


where $m_{3}$ is the largest neutrino mass eigenvalue. The recently improved $90 \%$ C.L. upper bound on $M_{e e}$ from $0 \nu \beta \beta$ decay experiments is 21]

$$
\left|M_{e e}\right|<0.2 \mathrm{eV}
$$

The GENIUS experiment is anticipated to be sensitive to $\left|M_{e e}\right|$ as low as $0.01 \mathrm{eV}$ [22].

Finally, since individual masses are not in general directly measurable, more appropriate variables are the sum of the neutrino masses, $\sum m_{\nu}$, and neutrino mass-squared differences. For three nearly degenerate neutrinos the sum of neutrino masses is approximately given by

$$
\sum m_{\nu} \simeq 3 m_{1}
$$

A measurement of the cosmological power spectrum from (i) the cosmic microwave background radiation by MAP [23] and PLANCK [24, (ii) red-shift surveys by the Sloan Digital Sky Survey (SDSS) [25] and the Two-Degree Field (2dF) [26], and (iii) the Ly $\alpha$ forest of neutral hydrogen absorption in quasar spectra [14] may be sensitive to $\sum m_{\nu}$ as low as $0.4 \mathrm{eV}$ [13.

3. Limits on the solar oscillation amplitude when $\sum \boldsymbol{m}_{\boldsymbol{\nu}}>\mathbf{0 . 4 5} \mathrm{eV}$. The neutrino mass eigenstates will be nearly degenerate if the mass eigenvalues

$$
m_{1} \simeq m_{2} \simeq m_{3}
$$

are large with respect to the mass splittings $\delta m_{j k}^{2}$. In this event, the mass splitting between the smallest and largest masses is

$$
m_{3}-m_{1}=\frac{\delta m_{31}^{2}}{\left(m_{3}+m_{1}\right)} \simeq \frac{\delta m_{a t m}^{2}}{2 m_{1}} \simeq \frac{0.0035 \mathrm{eV}^{2}}{2 m_{1}} .
$$

This mass difference is at least an order of magnitude smaller than $m_{1}$ for $m_{1}>0.15 \mathrm{eV}$, i.e., for $\sum m_{\nu}>0.45 \mathrm{eV}$. For such small mass splittings the $0 \nu \beta \beta$ decay limit in both Spectra I and II can be written

$$
\left|c_{1}^{2} c_{3}^{2}+c_{1}^{2} s_{3}^{2} e^{i \phi_{2}}+s_{1}^{2} e^{i \phi_{3}}\right|=\frac{\left|M_{e e}\right|}{m_{1}} \leq \frac{\left|M_{e e}\right|_{\max }}{m_{1}} .
$$

The left-hand side of Eq. (15) may be represented by the sum of three complex vectors whose directions (the phase angles) are unknown but whose lengths are determined by the mixing matrix parameters. A geometric interpretation of the constraint in Eq. (15) is that the longest side minus the sum of the two shorter sides must be less than $\left|M_{e e}\right|_{\max } / m_{1}$. Given the current limits on $s_{1}$, one of the two sides $c_{1}^{2} c_{3}^{2}$ or $c_{1}^{2} s_{3}^{2}$ must be the longest; without loss of generality, we assume $c_{3}>s_{3}$, so that $c_{1}^{2} c_{3}^{2}$ is the longest side. This limit is represented diagrammatically in Fig. 20 for both the small-angle and maximal-mixing solar solutions for two different values of $m_{1}: m_{1}=0.2 \mathrm{eV}$ (the current upper limit on $\left|M_{e e}\right|$ ) and $m_{1}=4.4 \mathrm{eV}$ (the upper limit on $m_{1}$ from tritium beta decay measurements [27, 28]).

Algebraically the constraint of Eq. (15) may be written

$$
c_{1}^{2} c_{3}^{2}-c_{1}^{2} s_{3}^{2}-s_{1}^{2} \leq \frac{\left|M_{e e}\right|_{\max }}{m_{1}} .
$$


Then using Eq. (12), the $0 \nu \beta \beta$ decay limit becomes

$$
s_{3}^{2} \geq \frac{1-2 s_{1}^{2}-\frac{3\left|M_{e e}\right|_{\max }}{\sum m_{\nu}}}{2 c_{1}^{2}} .
$$

This in turn implies that the solar $\nu_{e} \rightarrow \nu_{e}$ oscillation amplitude is constrained by

$$
A_{\text {sun }}^{e e} \equiv 4 c_{1}^{4} s_{3}^{2} c_{3}^{2} \geq 1-\left(\frac{3\left|M_{e e}\right|_{\max }}{\sum m_{\nu}}\right)^{2}-2 s_{1}^{2}\left(1+\frac{3\left|M_{e e}\right|_{\max }}{\sum m_{\nu}}\right) .
$$

The same result is obtained with $s_{3}>c_{3}$.

For any value of $\sum m_{\nu}>3\left|M_{e e}\right|_{\max } /\left(1-2 s_{1}^{2}\right)$ there will be a lower limit on the size of $A_{\text {sun }}^{e e}$ from Eq. (18); the most conservative limit occurs for the maximum value of $s_{1}^{2}$. In Fig. 3 we plot the lower limit on $A_{\text {sun }}^{e e}$ versus $\sum m_{\nu}$ for $\left|M_{e e}\right|_{\max }=0.2 \mathrm{eV}$ and the current upper bound of $s_{1}=0.3$. For large $\sum m_{\nu}$ the lower bound on $A_{\text {sun }}^{e e}$ approaches $1-2\left(s_{1}^{2}\right)_{\max } \simeq 0.82$.

Given the current upper bound on $\left|M_{e e}\right|$ from $0 \nu \beta \beta$ decay, Fig. 3 shows that for $\sum m_{\nu} \geq$ $0.75 \mathrm{eV}$, the small-angle matter-enhanced solar solution is excluded for three nearly degenerate Majorana neutrinos. If the upper bound on $s_{1}$ were to become more stringent, the limit in Eq. (18) would be tightened, and the small solar mixing solution would be excluded for $\sum m_{\nu}$ smaller than $0.75 \mathrm{eV}$. Vacuum solar solutions, which have $A_{\text {sun }}^{e e}=0.6-1.0$ [9, 10, 11], are allowed.

The $0 \nu \beta \beta$ constraint may be understood qualitatively as follows. If there is small mixing of $\nu_{e}$ with two of the mass eigenstates, then there is one dominant $U_{e j}$, in which case it is impossible to have the three contributions to $M_{e e}$ combine to give a small result for $\left|M_{e e}\right|$ if the individual neutrino masses are greater than $\left|M_{e e}\right|$; see Fig. 2a. On the other hand, with large-angle solar mixing $\nu_{e}$ is a roughly equal mixture of two eigenstates, and the three contributions to $M_{e e}$ can give a much smaller result. In fact, $M_{e e}=0$ is always possible if $s_{3}=c_{3}$ (maximal solar mixing); see Fig. 2 $\mathrm{b}$. Although a stringent upper bound on $\left|M_{e e}\right|$ does not rule out Majorana neutrinos when $s_{3}=c_{3}$, it does put a very tight limit on the Majorana phase angle $\phi_{2}$. This is illustrated in Fig. 2 $\mid \mathrm{b}$, where a very small $\left|M_{e e}\right|$ and small $s_{1}^{2}$ imply that the Majorana phase angle $\phi_{2}$ is close to $\pi$. Such a value for $\phi_{2}$ is a natural consequence of $C P$ conservation if $\nu_{1}$ and $\nu_{2}\left(\nu_{2}\right.$ and $\left.\nu_{3}\right)$ have opposite $C P$ eigenvalues in Spectrum I (II) [29].

4. Constraints on the neutrino mass spectrum for arbitrary $\sum \boldsymbol{m}_{\boldsymbol{\nu}}$. For $\sum m_{\nu}<$ $1 \mathrm{eV}$, the small splitting of the neutrino masses indicated by the atmospheric and solar experiments can affect the limit in Eq. (18). Writing Eqs. (8) and (9) in terms of $m_{1}$ and $\delta m_{\text {atm }}^{2}$, in the limit that $\delta m_{\text {sun }}^{2}$ can be ignored we find

$$
\begin{aligned}
M_{e e} & =c_{1}^{2} c_{3}^{2} m_{1}+c_{1}^{2} s_{3}^{2} m_{1} e^{i \phi_{2}}+s_{1}^{2} \sqrt{m_{1}^{2}+\delta m_{a t m}^{2}} e^{i \phi_{3}}, \\
\sum m_{\nu} & =2 m_{1}+\sqrt{m_{1}^{2}+\delta m_{\text {atm }}^{2}},
\end{aligned}
$$

in Spectrum I, and

$$
\begin{aligned}
M_{e e} & =c_{1}^{2} c_{3}^{2} \sqrt{m_{1}^{2}+\delta m_{\text {atm }}^{2}}+c_{1}^{2} s_{3}^{2} \sqrt{m_{1}^{2}+\delta m_{a t m}^{2}} e^{i \phi_{2}}+s_{1}^{2} m_{1} e^{i \phi_{3}}, \\
\sum m_{\nu} & =m_{1}+2 \sqrt{m_{1}^{2}+\delta m_{a t m}^{2}},
\end{aligned}
$$


for Spectrum II. Furthermore, the structure of the mass spectrum requires

$$
\begin{aligned}
& \sum m_{\nu}>\sqrt{\delta m_{a t m}^{2}}, \quad(\mathrm{I}), \\
& \sum m_{\nu}>2 \sqrt{\delta m_{a t m}^{2}}, \quad(\mathrm{II}) .
\end{aligned}
$$

(a) Small-angle solar mixing. For the small-angle solar solution (which has $2 \times 10^{-3} \leq$ $A_{\text {sun }}^{e e} \leq 10^{-2}$ [10 and thus $0.02 \leq s_{3} \leq 0.05$ ), $s_{3}^{2}$ is negligible in Eqs. (19) and (21); then the allowed ranges for $\left|M_{e e}\right|$ are

$$
\begin{gathered}
\left|c_{1}^{2} m_{1}-s_{1}^{2} \sqrt{m_{1}^{2}+\delta m_{a t m}^{2}}\right| \leq\left|M_{e e}\right| \leq c_{1}^{2} m_{1}+s_{1}^{2} \sqrt{m_{1}^{2}+\delta m_{a t m}^{2}}, \text { (I), } \\
c_{1}^{2} \sqrt{m_{1}^{2}+\delta m_{a t m}^{2}}-s_{1}^{2} m_{1} \leq\left|M_{e e}\right| \leq c_{1}^{2} \sqrt{m_{1}^{2}+\delta m_{a t m}^{2}}+s_{1}^{2} m_{1}, \text { (II). }
\end{gathered}
$$

The allowed bands for $\left|M_{e e}\right|$ are shown in Fig. Ha versus $\sum m_{\nu}$ (which is related to $m_{1}$ via Eqs. (20) and (22) ) in Spectra I and II for $\delta m_{\text {atm }}^{2}=3.5 \times 10^{-2} \mathrm{eV}^{2}$ and $0 \leq s_{1} \leq 0.18$ (the CHOOZ constraint for that $\delta m_{\text {atm }}^{2}$ ). Since both $s_{1}^{2}$ and $s_{3}^{2}$ are small in the small-angle solar solution, only one mass eigenstate contributes significantly to $M_{e e}$ and there is nearly a one-to-one correspondence between $\left|M_{e e}\right|$ and $m_{1}$ (and hence between $\left|M_{e e}\right|$ and $\sum m_{\nu}$ ):

$$
\begin{aligned}
& \left|M_{e e}\right| \simeq m_{1}, \quad(\mathrm{I}), \\
& \left|M_{e e}\right| \simeq \sqrt{m_{1}^{2}+\delta m_{a t m}^{2}}, \quad \text { (II) }
\end{aligned}
$$

This relation between $\left|M_{e e}\right|$ and $m_{1}$ for the small-angle solar solution implies that if a nonzero $\left|M_{e e}\right|$ is measured, all of the neutrino masses will be determined for either mass spectra in Fig. 11.

The current limit $\left|M_{e e}\right|<0.2 \mathrm{eV}$ in Fig. Ga shows that for $\sum m_{\nu}>0.75 \mathrm{eV}$ the smallangle solar solution is ruled out for Majorana neutrinos in both Spectra I and II. Although the range of allowed $\left|M_{e e}\right|$ versus $\sum m_{\nu}$ expands if the presently allowed range of $\delta m_{a t m}^{2}$ from the Super-K atmospheric data is used, the qualitative behavior of the allowed regions for Spectra I and II remains the same. Improved limits on $s_{1}$ and $\delta m_{\text {atm }}^{2}$ will shrink the allowed ranges for the small-angle solar solution. The value of $\delta m_{a t m}^{2}$ can be more precisely measured in the $\mathrm{K} 2 \mathrm{~K}$ [30] and MINOS [31] long-baseline experiments.

It is evident from Fig. 四 that future measurements of $\left|M_{e e}\right|$ and $\sum m_{\nu}$ could rule out the small-angle solar solution for Majorana neutrinos in one or both of the mass spectra possibilities. For example, Spectrum II is ruled out for any $\sum m_{\nu}$ when $\left|M_{e e}\right|<0.05 \mathrm{eV}$, and both spectra are excluded if, e.g., $\sum m_{\nu}>0.4 \mathrm{eV}$ and $\left|M_{e e}\right|<0.1 \mathrm{eV}$. Alternatively, nonzero measurements for both $\left|M_{e e}\right|$ and $\sum m_{\nu}$ could distinguish between the two mass spectra.

(b) Large-angle solar solution. For the large-angle vacuum or matter-enhanced solar solutions, the allowed range of $\left|M_{e e}\right|$ expands considerably. For the vacuum solar solution, the solar oscillation amplitude is large, and may be maximal. Vacuum solutions that allow maximal mixing can never be ruled out simply by lowering the limit on $\left|M_{e e}\right|$. However, it may still be possible to distinguish Spectrum I from II.

The largest possible $\left|M_{e e}\right|$ occurs when all terms in Eqs. (19) and (21) add in phase, and is given by the upper limits in Eqs. (25) and (26). Then given $\delta m_{a t m}^{2}$ and Eqs. (20) and (22), 
the largest possible $\left|M_{e e}\right|$ can be found for a given $\sum m_{\nu}$; these results are shown in Fig. $4 \mathrm{~b}$ for $\delta m_{a t m}^{2}=3.5 \times 10^{-3} \mathrm{eV}^{2}$. Figure $4 \mathrm{~b}$ shows that a nonzero measurement on $\left|M_{e e}\right|$ implies a lower limit on $\sum m_{\nu}$; for example, $\left|M_{e e}\right|=0.06 \mathrm{eV}$ implies that $\sum m_{\nu}>0.20(0.12) \mathrm{eV}$ in Spectrum I (II).

As can be seen in Fig. Ф $4 \mathrm{~b}$, there are certain values of $\left|M_{e e}\right|$ and $\sum m_{\nu}$ that are possible only for Spectrum I, and others that are possible only for II. In either of these cases a unique mass spectrum could be selected; this conclusion follows from the fact that $\sum m_{\nu}$ must always be greater than $2 \sqrt{\delta m_{\text {atm }}^{2}}$ in Spectrum II, whereas in I it can be less. Furthermore, since there are two larger masses in II and only one larger mass in I, $\left|M_{e e}\right|$ can be larger in II than in I for the same value of $\delta m_{a t m}^{2}$. There is also a large region in Fig. 碃 that could be obtained in either I or II, in which case the mass spectra would not be differentiated by the $0 \nu \beta \beta$ and $\sum m_{\nu}$ measurements. The allowed regions for $\left|M_{e e}\right|$ expand as $\delta m_{a t m}^{2}$ is varied over its presently allowed range, but there is still considerable area unique to each spectrum.

The allowed regions for large solar mixing are obtained without using any information about $\theta_{3}$. If $s_{3}=c_{3}$ (which corresponds to maximal solar mixing), then as noted above the lower bound on $\left|M_{e e}\right|$ is zero. A precise determination of $s_{3}$ could reduce the allowed ranges of $\left|M_{e e}\right|$ versus $\sum m_{\nu}$.

5. Summary. Our main conclusions regarding models with three Majorana neutrinos are as follows:

(i) For the small-angle matter-enhanced solar solutions there is an approximate relation between $\left|M_{e e}\right|$ and $\sum m_{\nu}$, which implies that a nonzero measurement of $\left|M_{e e}\right|$ determines $\sum m_{\nu}$ in these models. If the sum of neutrino masses is determined by cosmological power spectra measurements to be greater than about $0.75 \mathrm{eV}$, then the small-angle solar solution is ruled out by the current stringent limit on neutrinoless double beta decay. Further improvement of the $0 \nu \beta \beta$ decay limit could rule out the small-angle solar solution at even smaller nonzero values of $\sum m_{\nu}$, or perhaps distinguish between Spectrum I (in which the two lightest mass eigenstates are responsible for the solar oscillation) and Spectrum II (the two heaviest mass eigenstates are responsible for the solar oscillation).

(ii) Large-angle vacuum oscillation scenarios are largely safe from $0 \nu \beta \beta$ decay experimental constraints. However, a nonzero measurement of $\left|M_{e e}\right|$ places a lower limit on $\sum m_{\nu}$ in these models. The next generation of experiments measuring $\left|M_{e e}\right|$ and $\sum m_{\nu}$ may be able to distinguish between Spectra I and II, and could perhaps give information on relative $C P$ phases of the neutrino mass eigenstates. In particular, in models with maximal solar mixing, $\left|M_{e e}\right|$ substantially below $\sum m_{\nu} / 3$ is only possible when the two neutrino mass eigenstates primarily contributing to $M_{e e}$ have $C P$ phases that differ by about $\pi$.

Acknowledgements. This work was supported in part by the U.S. Department of Energy, Division of High Energy Physics, under Grants No. DE-FG02-94ER40817 and No. DEFG02-95ER40896, and in part by the University of Wisconsin Research Committee with funds granted by the Wisconsin Alumni Research Foundation. 


\section{References}

[1] Super-Kamiokande Collaboration, Y. Fukuda et al., Phys. Rev. Lett. 82, 1810 (1999); Phys. Rev. Lett. 82, 2430 (1999); M.B. Smy, hep-ex/9903034, talk at DPF-99, Los Angeles, California, January 1999; G. Sullivan, talk at Aspen Winter Conference, January, 1999.

[2] Super-Kamiokande Collaboration, Y. Fukuda et al., Phys. Rev. Lett. 81, 1562 (1998); Phys. Rev. Lett. 82, 2644 (1999).

[3] B.T. Cleveland et al., Nucl. Phys. B (Proc. Suppl.) 38, 47 (1995); GALLEX collaboration, W. Hampel et al., Phys. Lett. B 388, 384 (1996); SAGE collaboration, J.N. Abdurashitov et al., Phys. Rev. Lett. 77, 4708 (1996); Kamiokande collaboration, Y. Fukuda et al., Phys. Rev. Lett, 77, 1683 (1996);

[4] See, e.g., J.N. Bahcall, S. Basu, and M.H. Pinsonneault, Phys. Lett. B 433, 1 (1998), and references therein.

[5] Kamiokande collaboration, K.S. Hirata et al., Phys. Lett. B 280, 146 (1992); Y. Fukuda et al., Phys. Lett. B 335, 237 (1994); IMB collaboration, R. Becker-Szendy et al., Nucl. Phys. Proc. Suppl. 38 B, 331 (1995); Soudan-2 collaboration, W.W.M. Allison et al., Phys. Lett. B 391, 491 (1997); MACRO collaboration, M. Ambrosio et al., Phys. Lett. B 434, 451 (1998).

[6] G. Barr, T.K. Gaisser, and T. Stanev, Phys. Rev. D 39, 3532 (1989); M. Honda, T. Kajita, K. Kasahara, and S. Midorikawa, Phys. Rev. D 52, 4985 (1995); V. Agrawal, T.K. Gaisser, P. Lipari, and T. Stanev, Phys. Rev. D 53, 1314 (1996); T.K. Gaisser et al., Phys. Rev. D 54, 5578 (1996); T.K. Gaisser and T. Stanev, Phys. Rev. D 57, 1977 (1998).

[7] J.G. Learned, S. Pakvasa, and T.J. Weiler, Phys. Lett. B 207, 79 (1988); V. Barger and K. Whisnant, Phys. Lett. B 209, 365 (1988); K. Hidaka, M. Honda, and S. Midorikawa, Phys. Rev. Lett. 61, 1537 (1988).

[8] R. Barbieri, L.J. Hall, D. Smith, A. Strumia, and N. Weiner, hep-ph/9807235.

[9] V. Barger and K. Whisnant, hep/ph-9812273, to appear in Phys. Rev. D.

[10] J.N. Bahcall, P.I. Krastev, and A.Yu. Smirnov, Phys. Rev. D 58, 096016 (1998).

[11] V. Barger and K. Whisnant, hep-ph/9903262, to appear in Phys. Lett. B.

[12] See, e.g., J.R. Primack, Science 280, 1398 (1998); J.R. Primack and M.A.K. Gross, astro-ph/9810204; E. Gawiser and J. Silk, Science 280, 1405 (1998).

[13] D.J. Eisenstein, W. Hu, and M. Tegmark, Phys. Rev. Lett. 80, 5255 (1998); astro$\mathrm{ph} / 9807130$. 
[14] R.A.C. Croft, W. Hu, and R. Dave, astro-ph/9903335.

[15] M. Doi et al., Phys. Lett. B 102, 323 (1981).

[16] The Physics of Massive Neutrinos, B. Kayser, F. Gibrat-Debu, and F. Perrier (World Scientific, Singapore, 1989); Massive Neutrinos in Physics and Astrophysics, R. Mohapatra and P.B. Pal (World Scientific, Singapore, 1991).

[17] H. Minakata and O. Yasuda, Phys. Rev. D 56, 1692 (1997); T. Fukuyama, K. Matsuda, and H. Nishiura, Phys. Rev. D 57, 5844 (1998); H. Georgi and S.L. Glashow, hepph/9808293; G.C. Branco, M.N. Rebelo, and J.I. Silva-Marcos, Phys. Rev. Lett. 82, 683 (1999); R. Adhikari and G. Rajasekaren, hep-ph/9812361.

[18] L. Wolfenstein, Phys. Rev. D 17, 2369 (1978); V. Barger, R.J.N. Phillips, and K. Whisnant, Phys. Rev. D 22, 2718 (1980); P. Langacker, J.P. Leveille, and J. Sheiman, Phys. Rev. D 27, 1228 (1983); S.P. Mikheyev and A. Smirnov, Yad. Fiz. 42, 1441 (1985); Nuovo Cim. C 9, 17 (1986); S.J. Parke, Phys. Rev. Lett. 57, 1275 (1986); S.J. Parke and T.P. Walker, Phys. Rev. Lett. 57, 2322 (1986); W.C. Haxton, Phys. Rev. Lett. 57, 1271 (1986); T.K. Kuo and J. Pantaleone, Rev. Mod. Phys. 61, 937 (1989).

[19] V. Barger, R.J.N. Phillips, and K. Whisnant, Phys. Rev. D 24, 538 (1981); S.L. Glashow and L.M. Krauss, Phys. Lett. B 190, 199 (1987); V. Barger, R.J.N. Phillips, and K. Whisnant, Phys. Rev. Lett. 65, 3084 (1990); ibid. 69, 3135 (1992); A. Acker, S. Pakvasa, and J. Pantaleone, Phys. Rev. D 43, 1754 (1991); P.I. Krastev and S.T. Petcov, Phys. Lett. B 285, 85 (1992); Phys. Rev. D 53, 1665 (1996); N. Hata and P. Langacker, Phys. Rev. D 56, 6107 (1997); S.L. Glashow, P.J. Kernan, and L.M. Krauss, Phys. Lett. B 445, 412 (1999).

[20] CHOOZ collaboration, M. Apollonio et al., Phys. Lett. B 420, 397 (1998).

[21] L. Baudis et al., hep-ex/9902014.

[22] H.V. Klapdor-Kleingrothaus, J. Hellmig, and M. Hirsch, J. Phys. G 24, 483 (1998).

[23] See, e.g., http://map.gsfc.nasa.gov

[24] See, e.g., http://astro.estec.esa.nl/SA-general/Projects/Planck

[25] See, e.g., http://www.sdss.org

[26] See, e.g., http://meteor.anu.edu.au/ colless/2dF

[27] A.I. Belesev et al., Phys. Lett. B 350, 263 (1995); V.M. Lobashev et al., in Proc. of Neutrino-96, ed. by K. Enqvist, K. Huitu, and J. Maalampi (World Scientific, Singapore, 1997).

[28] V. Barger, T.J. Weiler, and K. Whisnant, Phys. Lett. B 442, 255 (1998). 
[29] $C P$ phases of Majorana neutrinos are discussed in Ref. [17]; see also L. Wolfenstein, Phys. Lett. B 107, 77 (1981); P.B. Pal and L. Wolfenstein, Phys. Rev. D 25, 766 (1982); K. Hagiwara and N. Okamura, hep-ph/9811495.

[30] K2K Collaboration, Y. Oyama, Proc. of the YITP Workshop on Flavor Physics, Kyoto, Japan, 1998, hep-ex/9803014.

[31] MINOS Collaboration, "Neutrino Oscillation Physics at Fermilab: The NuMI-MINOS Project," NuMI-L-375, May 1998. 
I

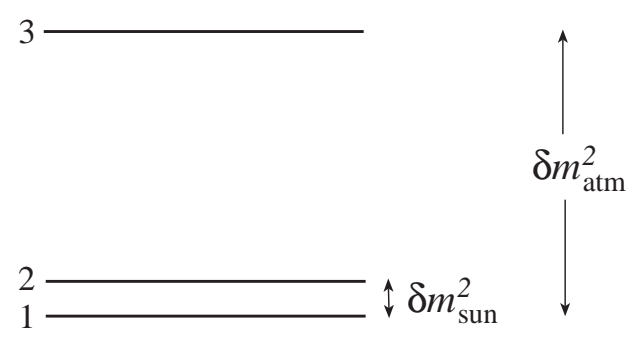

II
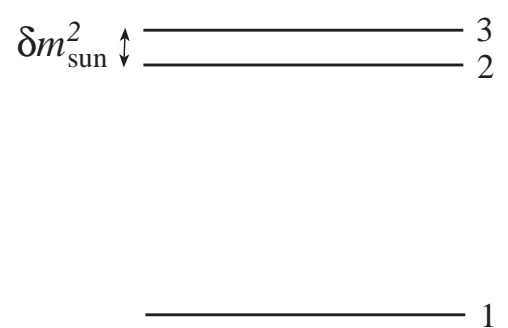

Figure 1: The two possibilites for the three-neutrino mass spectrum.

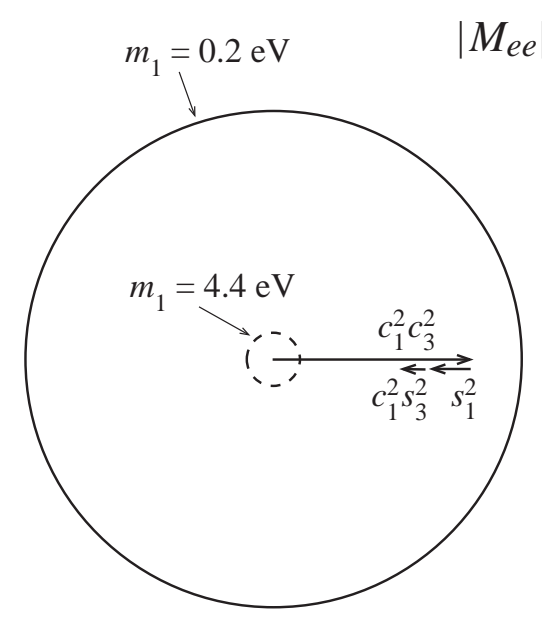

(a) Small angle solar

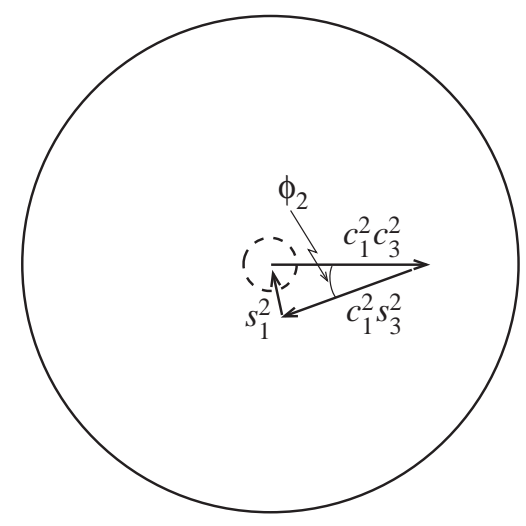

(b) Large angle solar

Figure 2: Typical examples of the $\left|M_{e e}\right| / m_{1}$ bound (with $\left|M_{e e}\right|<0.2 \mathrm{eV}$ ) for three nearly degenerate Majorana neutrinos for the (a) small-angle and (b) maximal-mixing solar solutions. The circles are the bounds assuming $m_{1}=0.2 \mathrm{eV}$ (solid) and $4.4 \mathrm{eV}$ (dashed), where $m_{1}=\sum m_{\nu} / 3$. In (a) the bound is satisfied for $m_{1}=0.2 \mathrm{eV}$ but not for $m_{1}=4.4 \mathrm{eV}$; in (b) the bound is satisfied in both $m_{1}$ cases for $\phi_{2} \approx \pi$. 


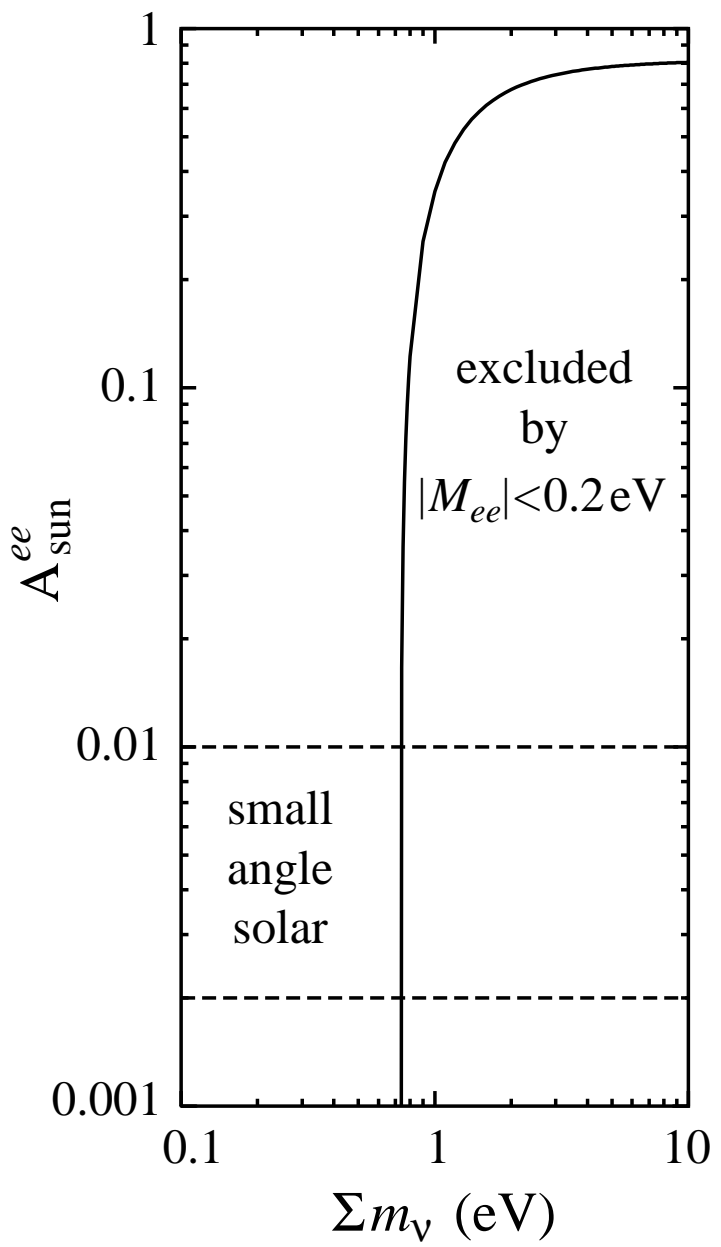

Figure 3: Lower bound on the solar oscillation amplitude $A_{\text {sun }}^{e e}$ versus the sum of the neutrino masses $\sum m_{\nu}$ based on the current bound from $0 \nu \beta \beta$ decay, $\left|M_{e e}\right|<0.2 \mathrm{eV}$. Spectra I and II give nearly identical results. The allowed range [10] for $A_{\text {sun }}^{\text {ee }}$ for the small-angle matterenhanced solar solution is also shown. 

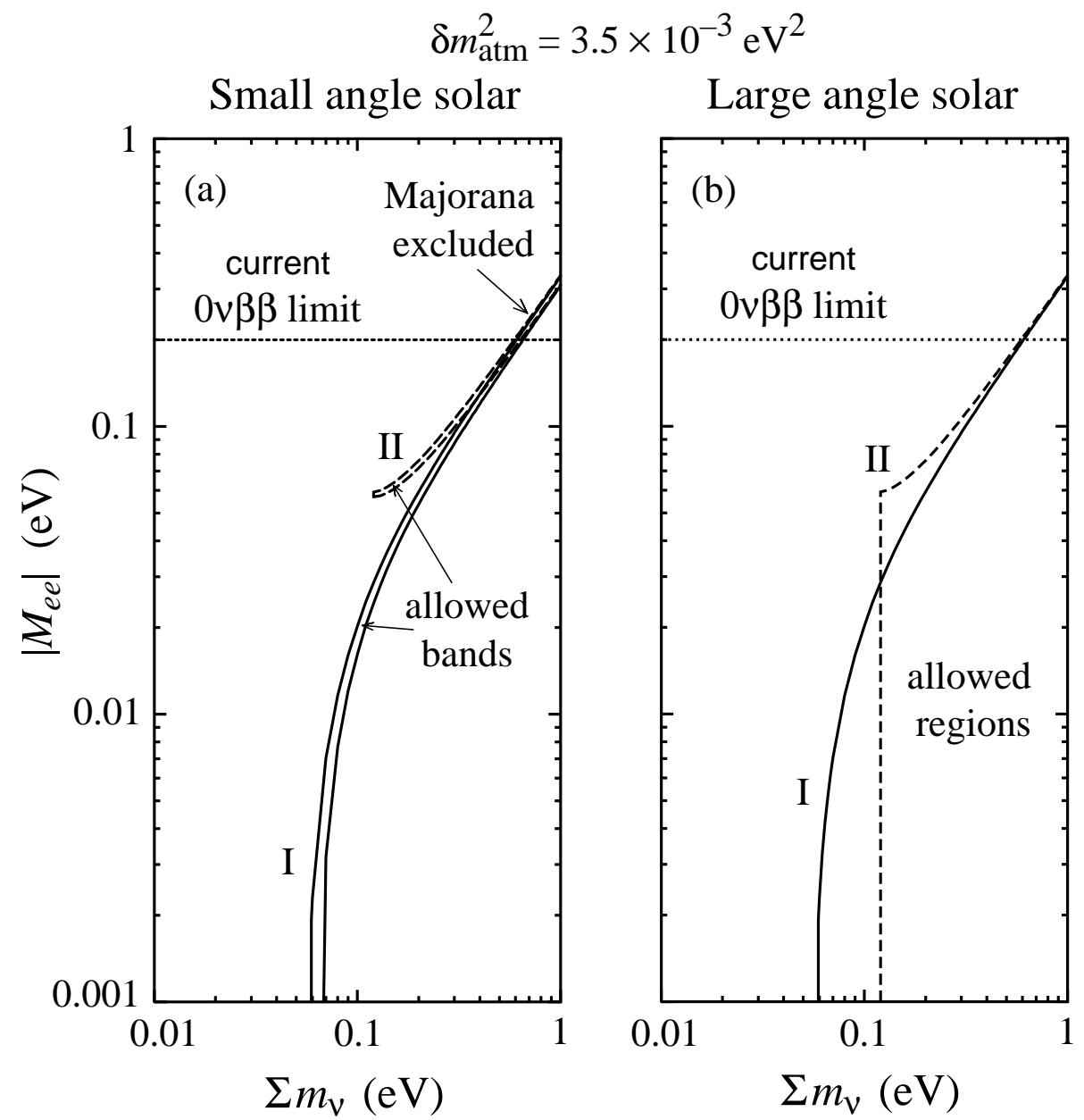

Figure 4: Allowed regions of $\left|M_{e e}\right|$ versus the sum of the neutrino masses $\sum m_{\nu}$ with $\delta m_{a t m}^{2}=3.5 \times 10^{-3} \mathrm{eV}^{2}$ for the (a) small-angle and (b) large-angle solar solutions. The mixing parameter $s_{1}$ has been varied over the values $0 \leq \sin \theta_{1} \leq 0.18$ allowed by the CHOOZ constraint [20]. Results are shown for Spectra I (solid curves) and II (dashed). 